\title{
Atividade antibacteriana do óleo essencial de Melampodium divaricatum (Rich.) DC., Asteraceae
}

\author{
Grace Priscila Pelissari, ,"1 Rosemeire Cristina Linhari Rodrigues Pietro, ${ }^{2}$ \\ Raquel Regina Duarte Moreira ${ }^{1}$
}

\author{
${ }^{1}$ Departamento de Princípios Ativos Naturais e Toxicologia, Faculdade de Ciências Farmacêuticas, \\ Universidade Estadual Paulista, 14801-902 Araraquara-SP, Brasil \\ ${ }^{2}$ Departamento de Fármacos e Medicamentos, Faculdade de Ciências Farmacêuticas, \\ Universidade Estadual Paulista, 14801-902 Araraquara-SP, Brasil.
}

\begin{abstract}
RESUMO: Melampodium divaricatum (Rich) DC., Asteraceae é uma espécie encontrada na América Latina sendo no Brasil principalmente na região Nordeste. Popularmente vem sendo empregada como antiinflamatória e cicatrizante e dentre vários componentes químicos relatados na literatura, destaca-se a presença de óleos essenciais em suas partes aéreas. Considerando o potencial antimicrobiano in vitro e in vivo de óleos essenciais presentes em muitas plantas e do amplo potencial farmacológico destes, este trabalho teve como objetivo avaliar a atividade antibacteriana do óleo essencial obtido a partir de partes aéreas de $M$. divaricatum utilizando-se o método de difusão em disco frente às bactérias Staphylococcus aureus (ATCC 25923), Bacillus subtilis (ATCC 9372), Escherichia coli (ATCC 25922), Proteus mirabilis (ATCC 25933) e as cepas de campo: Pseudomonas aeruginosa, Shigella sonnei, Serratia marcescens. De acordo com os resultados obtidos no presente trabalho, apenas as espécies Gram-positivas ( $S$. aureus e B. subtilis) mostraramse sensíveis ao óleo essencial, e as demais espécies testadas não tiveram seu crescimento inibido.
\end{abstract}

Unitermos: Melampodium divaricatum, Asteraceae, óleo essencial, atividade antibacteriana.

\begin{abstract}
Antibacterial activity of the essential oil of Melampodium divaricatum (Rich.) DC, Asteraceae". Melampodium divaricatum is a species found in Latin America and in Brazil mainly in the Northeast region. It is popularly used as anti-inflammatory and cicatrizing and among several chemical compounds it can be noteworthy the presence of essential oils in aerial parts. Considering the antimicrobial potential of essential oils in vitro and in vivo as well as its ample pharmacologic potential the objective of this work was to evaluate the antibacterial activity of the crude essential oil obtained from the aerial parts of $M$. divaricatum by the method of disc diffusion against Staphylococcus aureus (ATCC 25923), Bacillus subtilis (ATCC 9372), Escherichia coli (ATCC 25922), Proteus mirabilis (ATCC 25933), the clinical strains Pseudomonas aeruginosa, Shigella sonnei and Serratia marcescens. Only the gram-positive species $S$. aureus and B. subtilis showed sensitivity to the essential oil and the other analyzed strains did not have growth inhibited.
\end{abstract}

Keywords: Melampodium divaricatum, Asteraceae, essential oil, antibacterial activity.

\section{INTRODUÇÃO}

Um dos maiores problemas de Saúde Pública, enfrentados nas últimas décadas foi o agravamento da resistência à antimicrobianos em populações bacterianas, principalmente de origem hospitalar (Huovinen \& Cars, 1998; Okeke et al., 1999; Oliveira et al., 2006).

Atualmente, registra-se um aumento significativo na freqüência do isolamento de bactérias que eram reconhecidamente sensíveis às drogas rotineiramente usadas na clínica, mas que se apresentam resistentes a quase todos os fármacos disponíveis no mercado, como descrito para várias bactérias multi-droga-resistentes
(Shiomori et al., 2002; Sakagami \& Kajamura, 2006).

Tal fato torna-se relevante, pois é crescente o número de pacientes em hospitais com a imunidade suprimida e com isso, novas infecções poderão ocorrer resultando em elevada morbidade e mortalidade (Nascimento et al., 2000; Caetano et al., 2002).

$\mathrm{O}$ Brasil possui um número muito grande de espécies vegetais consideradas medicinais as quais são muito utilizadas pela população para diversos problemas de sáude (Mors et al., 2000; Barbosa-Filho et al., 2005; Brandão et al., 2006). Porém, muitas destas plantas ainda não tiveram qualquer avaliação científica do seu uso medicinal, o que é essencial para que possam continuar a 
serem utilizadas com garantia de eficácia e segurança pela população.

Melampodium divaricatum é uma planta muito utilizada no Brasil, principalmente no Nordeste. Conhecida popularmente como falsa-calêndula, flor-amarela, estrelinha, flor-de-ouro (Lorenzi, 2000); picão-da-praia (Agra et al., 2007); fel-da-terra e salsa-da-praia (Botsaris, 2007) é comumente empregada na medicina popular (Girón et al., 1991). O chá de suas folhas e inflorescências, bem como da planta inteira tem sido empregado popularmente como cicatrizante, diaforético (Rocha Neto \& Faria, 2001), diurético e no tratamento da leucorréia (Agra et al., 2007). É uma planta anual, ereta, herbácea, ramificada, de 80$160 \mathrm{~cm}$ de altura, nativa da América do Sul. Trata-se de uma espécie daninha medianamente freqüente em quase todo o país, onde infesta principalmente lavouras anuais, pomares, cafezais e beira de estradas (Lorenzi, 2000).

Sob o ponto de vista químico $M$. divaricatum apresenta flavonóides (canferol e quercetina) (Hüther et al., 1999), cumarinas (Borges-del-Castillo et al., 1984), alcalóides pirrolizidínicos (Schüngel \& Passreiter, 2000), bem como compostos comumente presentes em óleos essenciais como o cariofileno, espatulenol e guaiacol (Hubert \& Wiemer, 1985).

Os óleos essenciais há muito tempo têm sido empregados popularmente por suas diversas propriedades terapêuticas, destacando-se: atividade antiviral, antiespasmódica, analgésica, antimicrobiana, cicatrizante, expectorante, relaxante, anti-séptica das vias respiratórias, larvicida, vermífuga e antiinflamatória (Costa et al., 2005; Oyedji \& Afolayan, 2006; Lima et al., 2006).

O presente trabalho representa o primeiro estudo do óleo essencial de $M$. divaricatum e teve como objetivo avaliar seu potencial antibacteriano in vitro.

\section{MATERIAL E MÉTODOS}

\section{Material vegetal}

Partes aéreas de Melampodium divaricatum (Rich.) DC., Asteraceae, foram coletadas nos meses de janeiro a março de 2007 no Horto de Plantas Medicinais e Tóxicas da Faculdade de Ciências Farmacêuticas da UNESP, Campus de Araraquara-SP, entre as coordenadas $21^{\circ} 48^{\prime} 51,4$ "'S e $48^{\circ} 12^{\prime} 5,1^{\prime \prime} \mathrm{W}$, na altitude de $661 \mathrm{~m}$. A exsicata da planta está depositada no Herbário do Instituto de Biociências da Unesp de Rio Claro-SP sob o número de registro HRCB 35294. O material vegetal foi coletado, selecionado e seco em estufa com circulação de ar e aquecimento a $40^{\circ} \mathrm{C}$.

\section{Microrganismos}

Sete cepas bacterianas foram utilizadas no controle de sensibilidade dos ensaios: Staphylococcus aureus (ATCC 25923), Bacillus subtilis (9372), Escherichia coli
(ATCC 25922), Proteus mirabilis (ATCC 25933), as cepas de campo Pseudomonas aeruginosa, Shigella sonnei e Serratia marcescens.

\section{Obtenção dos óleos essenciais}

Os óleos essenciais utilizados neste trabalho foram obtidos pelo processo de hidrodestilação utilizandose o aparelho de Clevenger modificado (Wasicky \& Akisue, 1969) durante $4 \mathrm{~h}$. e o seu rendimento foi calculado e expresso em $\%(\mathrm{p} / \mathrm{p})$.

\section{Avaliação da atividade antibacteriana}

O método empregado foi o de difusão em ágar baseado na norma M2-A8 do Clinical and Laboratory Institute (CLSI) com algumas modificações (NCCLS, 2003), utilizando discos de papel de filtro esterilizados de $6,0 \mathrm{~mm}$ de diâmetro aplicados sobre placas de Petri contendo ágar Müeller- Hinton. Os inóculos foram obtidos a partir de culturas dos microrganismos incubados a 35 ${ }^{\circ} \mathrm{C}$ por $24 \mathrm{~h}$.), preparando-se suspensões padronizadas equivalentes a escala 0,5 de McFarland $\left(10^{8} \mathrm{UFC} / \mathrm{mL}\right)$. Alíquotas de $100 \mu \mathrm{L}$ de cada suspensão bacteriana foram semeadas por superfície em placas de Petri contendo cerca de $15 \mathrm{~mL}$ do meio Mueller- Hinton, com uma espessura de aproximadamente $4 \mathrm{~mm}$ (Lennette, 1980).

Diferentes discos foram impregnados com alíquotas de $10 \mu \mathrm{L}$ de óleo essencial puro e $10 \mu \mathrm{L}$ de óleo essencial com adição do agente emulsificador Tween 80 $(0,5 \%)$, visando melhorar a difusão do óleo essencial no ágar. Como controle positivo utilizou-se $50 \mu \mathrm{L}$ de solução de ampicilina $(50 \mu \mathrm{g} / \mathrm{mL})$ e como controle negativo $10 \mu \mathrm{L}$ do agente emulsificador Tween $80(0,5 \%)$. As culturas com os discos foram incubadas a $35^{\circ} \mathrm{C}$ por $24 \mathrm{~h}$. Os resultados, dos ensaios realizados em triplicata, foram obtidos através da mensuração do diâmetro dos halos de inibição formados ao redor dos discos (NCCLS, 2003) e calculados pela média aritmética \pm desvio-padrão dos diâmetros, expressos em mm.

\section{Análise estatística}

As diferenças significativas entre as médias foram determinadas pelo teste de Tukey com nível de significância de $5 \%(\mathrm{p}<0,05)$.

\section{RESULTADOS E DISCUSSÃO}

Diversos estudos demonstram que os óleos essenciais apresentam atividade antibacteriana (Carvalho Jr et al, 2004; Mevy et al, 2007) e que sua atividade está intimamente relacionada a composição química refletindo nos possíveis mecanismos de ação destes frente às diferentes cepas bacterianas (Carson et al., 2002).

O rendimento do óleo essencial obtido a partir das 
partes aéreas secas de $M$. divaricatum foi $0,10 \pm 0,02 \%$, indicando um baixo rendimento o que é comumente observado na maioria das espécies medicinais (Wagner et al.,1984).

Visando melhorar a qualidade das análises das propriedades antimicrobianas com óleos essenciais, tornou-se comum a utilização de solventes, detergentes, ou agentes emulsificadores dentre eles o tween 80 , para facilitar a dispersão dos mesmos no meio de cultura (Bruni et al., 2004). Entretanto podem ocorrer interações do agente emulsificador com a substância teste, bem como o mesmo pode possuir atividade antibacteriana. Esses efeitos podem ser acentuados ou minimizados dependendo do modo de preparo da solução óleo-agente emulsificador (Hammer et al., 1999). Para minimizar esses efeitos, alguns autores propõem que os emulsificadores sejam utilizados em concentrações que variem entre 0,5 a $20 \%$ em solução com óleo. No presente estudo foi testado o óleo essencial na ausência ou presença do agente emulsificador, para comparação dos resultados obtidos para o óleo essencial de $M$. divaricatum frente às bactérias testadas (Tabela 1).

Os resultados obtidos no presente trabalho demonstraram a atividade antibacteriana in vitro do óleo essencial, pois houve inibição do crescimento das cepas Gram-positivas (S. aureus e B. subtillis), embora sem inibição do crescimento das cepas Gram-negativas testadas.

Resultados semelhantes aos observados em nosso estudo foram obtidos a partir do óleo essencial da espécie Cordia verbenacea, conhecida popularmente como ervabaleeira, onde as bactérias que se mostraram mais sensíveis foram as Gram-positivas, enquanto as Gram-negativas mostraram-se resistentes (Carvalho Jr et al., 2004).

Neste trabalho as culturas de $S$. aureus apresentaram frente ao óleo essencial puro ou na presença de tween halos de inibição 66,7 e 92,6\% menores que a ampicilina, respectivamente. Para B. subtilis apresentaram valores superiores à ampicilina de 41,6 e $66,7 \%$ para o óleo essencial puro e óleo essencial na presença de Tween 80. As outras bactérias testadas não foram inibidas pelo óleo essencial de $M$. divaricatum, assim como também o controle de Tween 80 não inibiu nenhuma bactéria na concentração testada.

Segundo estudo realizado por Cimanga et al. (2002) onde se avaliou a atividade antimicrobiana de óleos essenciais de diversas espécies vegetais dentre elas Eucalyptus globulus, Cymbopogon citratus, Ocimum americanum e Monodora myristica, a atividade antimicrobiana variou bastante de espécie para espécie, graças à infinidade e variabilidade de compostos químicos presentes, embora de forma geral os óleos essenciais tenham sido ativos principalmente contra os microrganismos Gram-positivos. Esses resultados podem estar diretamente relacionados com a estrutura da parede celular, tendo em vista que esta é a principal característica que diferencia os dois grupos bacterianos, onde a presença da membrana externa das cepas Gram-negativas acaba atuando como barreira para certos tipos de antibióticos, enzimas digestivas, detergentes e metais pesados (Tortora et al., 2003), e poderia estar impedindo a ação do óleo essencial, justificando assim os resultados observados neste estudo com o óleo essencial de $M$. divaricatum.

Diante da grande variedade na composição química dos óleos essenciais o seu mecanismo de ação pode envolver vários alvos na célula bacteriana (Burt, 2004). A maioria dos autores consideram a lipofilia de seus constituintes como a propriedade que explicaria a atividade antimicrobiana, característica que permitiria a partição destes compostos nos lipídeos da membrana celular e da mitocôndria, aumentando sua permeabilidade e levando ao extravasamento do conteúdo celular (Cowan, 1999). Outros autores sugerem que os componentes dos óleos essenciais também podem agir nas proteínas celulares localizadas nas membranas citoplasmáticas, entre elas as ATPases, através de sua acumulação na dupla camada lipídica e conseqüente destruição da interação lipídeoproteína. Possivelmente uma interação direta de compostos lipofílicos com porções hidrofóbicas das proteínas também pode acontecer (Sikkema et al., 1995).

Portanto, devido ao grande número e variedade dos compostos químicos presentes nos óleos essenciais, provavelmente sua atividade antimicrobiana não pode ser atribuída a um mecanismo de ação específico (Carson et al., 2002).

Tabela 1. Halos de inibição $(\mathrm{mm})$ pelo método de difusão em ágar na análise do óleo essencial obtido a partir de partes aéreas de Melampodium divaricatum.

\begin{tabular}{lcccc}
\hline Microrganismos & A & OP & OT & CT \\
\hline Staphylococcus aureus & $27 \pm 3^{\mathrm{a} *}$ & $18 \pm 4^{\mathrm{b}}$ & $25 \pm 3^{\mathrm{a}}$ & - \\
Bacillus subtilis & $12 \pm 2^{\mathrm{c}}$ & $17 \pm 3^{\mathrm{d}}$ & $20 \pm 2^{\mathrm{d}}$ & - \\
Pseudomonas & $11 \pm 1^{\mathrm{c}}$ & - & - & - \\
aeruginosa & & & & \\
Escherichia coli & $10 \pm 1 \mathrm{c}$ & - & - & - \\
Serratia marcescens & $10 \pm 1^{\mathrm{c}}$ & - & - & - \\
Shigella sonnei & $10 \pm 1^{\mathrm{c}}$ & - & - & - \\
Proteus mirabilis & $10 \pm 1^{\mathrm{c}}$ & - & - & - \\
\hline
\end{tabular}

A: Ampicilina; OP: Óleo essencial puro; OT: Óleo essencial com adição de Tween 80 (0,5\%); CT: controle - tween 80 (0,5\%) em água; : ausência de atividade (halos $\leq 7 \mathrm{~mm}$ ).

* Médias seguidas pela mesma letra, não diferem entre si, pelo teste de Tukey, com nivel de significância de $5 \%$

\section{CONCLUSÃO}

De acordo com os resultados obtidos neste trabalho pode-se concluir que o óleo essencial obtido a partir de partes aéreas de $M$. divaricatum apresenta potencial antibacteriano, especificamente contra as bactérias Gram-positivas e ausência de atividade contra bactérias Gram-negativas pelo método de difusão em ágar. Outras metodologias podem ser utilizadas com o intuito 
de corroborar com os resultados obtidos neste estudo, dentre elas a determinação da Concentração Inibitória Mínima. Posteriormente deveria ser realizado também um estudo químico do óleo essencial obtido a partir de partes aéreas de $M$. divaricatum visando a identificação dos seus principais componentes. Cabe ressaltar a importância de estudos posteriores para a determinação do mecanismo de ação do óleo essencial frente às bactérias testadas, o que poderá resultar em um novo agente antibacteriano.

\section{AGRADECIMENTOS}

Ao Luís Eduardo do Santos pelo apoio técnico e ao CNPq, pela bolsa de Mestrado concedida.

\section{REFERÊNCIAS}

Agra MF, França PF, Barbosa-Filho JM 2007. Synopsis of the plants known as medicinal and poisonous in Northeast of Brazil. Rev Bras Farmacogn 17: 114-140.

Barbosa-Filho JM, Vasconcelos THC, Alencar AA, Batista LM, Oliveira RAG, Guedes DN, Falcão HS, Moura MD, Diniz MFFM, Modesto-Filho J 2005. Plants and their active constituents from South, Central, and North America with hypoglycemic activity. Rev Bras Farmacogn 15: 392-413.

Borges-del-Castillo J, Martinez-Martir AI, Rodriguez-Luis F, Rodriguez-Ubis JC, Vazquez-Bueno P 1984. Isolation and syntesis of two coumarins from Melampodium divaricatum. Phytochemistry 23: 859-861.

Botsaris AS 2007. Plants used traditionally to treat malaria in Brazil: the archives of Flora Medicinal. J Ethnobiol Ethnomed 3: 1-8.

Brandão MGL, Cosenza GP, Moreira RA, Monte-Mor RLM 2006. Medicinal plants and other botanical products from the Brazilian Official Pharmacopoeia. Rev Bras Farmacogn 16: 408-420.

Bruni R, Medici A, Andreotti E, Fantin C, Muzzoli M, Dehesa M, Romagnoli C, Sacchetti G 2004. Chemical composition and biological activities of Ishpingo essential oil, a traditional Ecuadorian spice from Ocotea quixos (Lam.) Kosterm (Lauraceae) flower calices. Food Chem 85: 415-421.

Burt S 2004. Essential oils: their antibacterial properties and potential applications in foods - a review. Int J Food Microbiol 94: 223-253.

Caetano N, Saraiva A, Pereira R, Carvalho D, Pimentel MCB, Maia MBS 2002. Determinação de atividades antimicrobiana de extratos de plantas de uso popular como antiflamatório. Rev Bras Farmacogn 12 (Supl.): 132-135.

Carson CF, Mee BJ, Riley TV 2002. Mechanism of action of Melaleuca alternifolia (tea tree) oil on Staphylococcus aureus determined by time-kill, lysis, leakage and salt tolerance assays and electron microscopy. Antimicrob Agents Chemother 46: 1914-1920.

Carvalho Jr. PM, Rodrigues RF, Sawaya AC, Marques, MO, Shimizu MT, 2004. Chemical composition and antimicrobial activity of the essential oil of Cordia verbenaceae D.C. J Ethnopharmacol 95: 297-301.

Costa JGM, Rodrigues FFG, Angélico EC, Silva MR, Mota
ML, Santos NKA, Cardoso ALH, Lemos TLG 2005. Estudo químico-biológico dos óleos essenciais de Hyptis martiusii, Lippia sidoides e Syzigium aromaticum frente às larvas do Aedes aegypti. Rev Bras Farmacogn 15: 304-309.

Cowan MM 1999. Plant products as antimicrobial agents. Clin Microbiol Rev 12: 564-582.

Girón LM, Freire V, Alonzo A, Cáceres A 1991.Ethnobotanical survey of the medicinal flora used by the Caribs of Guatemala. J Ethnopharmacol 34: 173-187.

Hammer KA, Carson CF, Riley TV 1999. Antimicrobial activity of essential oils and others plants extracts. J Appl Microbiol 86: 985-990.

Hubert TD, Wiemer DF 1985. Ant-repellent terpenoids from Melampodium divaricatum. Phytochemistry 24: 11971198.

Huovinen P, Cars O 1998. Control of antimicrobial resistance: time for action. Braz Med J 317: 613-615.

Hüther J, Passreiter CM, Wray V, Willuhn G 1999. Acylated 2-hydroxythymol 3-O-diglycosides from Melampodium divaricatum. Phytochemistry 51: 979-986.

Lennette E 1980. Manual of clinical microbiology. 3ed. Washington: Americam Society for Microbiology.

Lima, IO, Oliveira RAG, Lima EO, Farias NMP, Souza EL 2006. Atividade antifúngica de óleos essenciais sobre espécies de Candida. Rev Bras Farmacogn 16: 197-201

Lorenzi H 2000. Plantas daninhas do Brasil: terrestres, aquáticas, parasitas e tóxicas. $3^{\mathrm{a}}$ ed.: Nova Odessa, SP: Instituto Plantarum.

Mevy JP, Bessiere JM, Dherbomez M, Millogo J, Viano J 2007. Chemical composition and some biological activities of the volatile oils of a chemotype of Lippia chevalieri Moldenke. Food Chem 101: 682-685.

Mors WB, Rizzini CT, Pereira NA 2000. Medicinal plants of Brazil. Michigan: Reference Publications Inc.

Nascimento GGF, Locatelli J, Freitas PC Silva GL 2000. Antibacterial activity of plant extracts and phytochemicals on antibiotic-resistant bacteria. Braz J Microbiol 31: 247256.

NCCLS (Clinical and Laboratory Standards Institute) 2003. Performance standards for antimicrobial disk susceptibility tests: approved standard. NCCLS document M2-A8-Wayne, Pennsylvania.

Okeke IN, Lamikanra A, Edelman R 1999. Socioeconomic and behavioral factors leading to acquired bacterial resistance to antibiotics in developing countries. Emerg Infect Dis 5: $18-27$.

Oyedji OA, Afolayan AJ 2006. Chemical composition an antibacterial activity of essential oil of the South African Mentha longifolia. J Essent Oil Res 18: 57-59.

Oliveira RAG, Lima EO, Vieira WL, Freire KRL, Trajano VN, Lima, IO, Souza EL, Toledo MS, Silva-Filho RN 2006a. Estudo da interferência de óleos essenciais sobre a atividade de alguns antibióticos usados na clínica. Rev Bras Farmacogn 16: 77-82.

Sakagami Y, Kajamura K 2006. Bactericidal activities of desinfectants against vancomycin-resistant Enterococci. $J$ Hosp Infect 56: 140-144.

Schüngel J, PassreiterCM2000. New diacylated 2-hydroxythymol derivatives from Melampodium divaricatum. $Z$. Naturforsch C 57: 966-968.

Shiomori T, Miyamoto H, Makashima K, Yoshida M, Fujiyoshi T, Udaka T, Inaba T, HirakiN2002. Evaluation of bedmaking- 
related airborne and surface methicillinresistant Staphylococcus aureus contamination. J Hosp Infect 50: 30-35.

Sikkema J, De Bont JAM, Poolman B 1995. Mechanisms of membrane toxicity of hydrocarbons. Microbiol Rev 59: 201-222.

Tortora GJ, Funke BR, Case CL 2003. Microbiologia. Porto Alegre: ArtMed.

Wagner HM, Bladt S, Zgainski EM 1984. Plant drug analysis. Berlin: Springer.

Wasiky R, Akisue G 1969. Um novo aparelho aperfeiçoado para extração de óleos essenciais. Rev Fac Farm Bioquim USP 7: 399-405. 